\title{
BEAM QUALITY MEASUREMENT OF 100-MeV TEST LINAC *
}

\author{
H. S. Kang", J.-Y. Choi, S. H. Nam, and S. S. Chang \\ Pohang Accelerator Laboratory, Pohang, Kyungbuk 790-784, Korea
}

\begin{abstract}
The $100-\mathrm{MeV}$ Test Linac was constructed for the high brightness applications such as free electron lasers and coherent radiation generation experiments at PAL. The linac consists of a thermionic RF-gun, an alpha magnet, two accelerating structures, and a beam analysing magnet. The RF-gun is a one-cell cavity with a tungsten dispenser cathode of 3-mm diameter, and the longitudinal matching from the RF-gun to the first accelerating column is done by the alpha magnet. The $\mathrm{RF}$ frequency is $2856 \mathrm{MHz}$, and a SLAC 5045 klystron feeds RF power to two accelerating columns and the RFgun. In the initial beam test the achieved beam energy and current were $75 \mathrm{MeV}$ and $50 \mathrm{~mA}$, respectively. The longitudinal acceptance measurement with the alpha magnet is done to minimize energy spread, and the bunch length was measured with a streak camera by using OTR (Optical Transition Radiation). In this paper, the beam quality measurement results are described in detail together with the detailed description of the machine.
\end{abstract}

\section{INTRODUCTION}

An electron linac with a design beam energy of 100 $\mathrm{MeV}$ has been prepared for new R\&D activities such as FEL (free electron laser) and SASE (self-amplified spontaneous emission) experiments at PAL (Pohang Accelerator Laboratory). New experiments like SASE require a high-brightness electron source; this implies a high peak current (10 A to $2000 \mathrm{~A}$ ) and a low transverse emittance (2 to $80 \mathrm{~mm}-\mathrm{mrad}$ ) [1]. Emittance and peak current requirements for infrared-FEL is not stringent compared to SASE.

Thermionic RF-gun was chosen as an electron beam source for the Test Linac because it is quite compact and relatively inexpensive source for high brightness applications in spite of some disadvantages such as a large energy spread and a short cathode life time. For SASE experiment the RF-gun will be replaced with photocathode RF-gun in the near future. The linac incorporates an alpha magnet for longitudinal phase matching from the gun to the first accelerating column because thermionic RF-gun inherently generates an electron beam with a large momentum spread.

"Work supported by the Ministry of Science and Technology, Korea

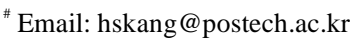

In the beam test of 1998 the achieved beam energy and current were $75 \mathrm{MeV}$ and $50 \mathrm{~mA}$, respectively. The beam loss was very large, about $50 \%$, at the first accelerating column [2]. Focusing solenoid coils were installed at the first accelerating column to reduce beam loss. The longitudinal acceptance measurement with the alpha magnet was done to minimize energy spread and beam loss. Bunch length was measured with a streak camera by using OTR (Optical Transition Radiation). Dependence of bunch length on field gradient of the alpha magnet was measured.

\section{MACHINE DESCRIPTION}

The linac consists of a thermionic RF-gun, an alpha magnet, two quadrupole doublets, two SLAC-type accelerating columns (AC\#1 and $\mathrm{AC \# 2}$ ), a quadrupole triplet (QT), and a beam analysing magnet as shown in Figure 1. There are three beam current monitors (BCM) and three beam profile monitors (BPRM) for beam instrumentation. There is an experimental area at the end of linac for FEL and SASE experiments, which has a dimension of 5-m width and 7-m length. A 2-m long drift space is reserved between $\mathrm{AC \# 1}$ and $\mathrm{AC \# 2}$ to insert a magnetic bunching system and a bending magnet for FEL research.

The RF-gun is a one-cell cavity with a tungsten dispenser cathode of 3-mm diameter [3]. The RF frequency is $2856 \mathrm{MHz}$, and a SLAC 5045 klystron feeds RF power to two accelerating columns and the RFgun. The maximum field gradient of the alpha magnet is $200 \mathrm{G} / \mathrm{cm}$. Electron moves along a $\alpha$-shaped trajectory in the alpha magnet, and the resultant bend angle is $278.6^{\circ}$. Two quadrupole doublets are used to focus the electron beam in the transport line from the gun to the first accelerating column. OTR bunch monitor chamber was installed at the linac end. The target is $2-\mathrm{mm}$ thick plate of stainless steel, from which OTR emits when the accelerated electron beam strikes on it, and the surface was mirror-finished.

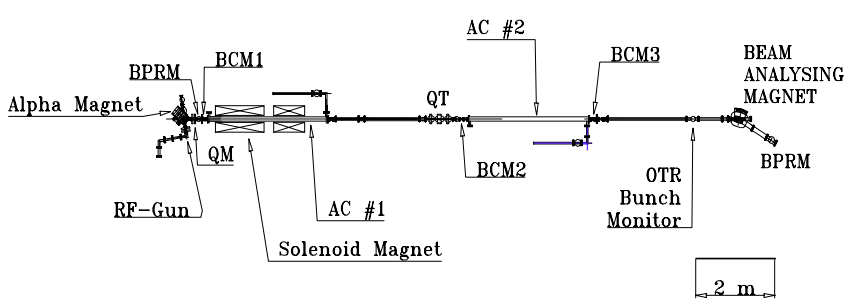

Figure 1: Layout of Test Linac. 


\section{BEAM QUALITY MEASUREMENTS}

\subsection{Beam Transmission and Energy Spread}

The beam transmission rate was improved up to $72 \%$ by the solenoid field focusing. Table 1 shows the measured data as the alpha magnet current changes. BCM\#1 measures beam current after the alpha magnet, and BCM\#3 measures beam current at the end of linac. Transmission rate is determined by longitudinal and transversal matching. Though the injected beam to the first accelerating column is high at 7-A current setting, the beam loss is very large because the transversal beam quality is very poor. Optimum matching condition is obtained at the current setting of $11.5 \mathrm{~A}$. The field gradient of alpha magnet at this current is 90.8 Gauss/cm.

The nominal RF power of the klystron is $30 \mathrm{MW}$ at the experiment, and the RF power fed to one accelerating column and the RF-gun were $12 \mathrm{MW}$ and 2.0 MW, respectively. The RF pulse width is $1.2 \mu \mathrm{s}$ and pulse repetition rate is $12 \mathrm{~Hz}$.

The energy and energy spread were also measured as the alpha magnet current changed. Output beam energy increases as the field gradient of alpha magnet increases. Measured energy spread is $\pm 1 \%$ at the beam loading of $100 \mathrm{~mA}$. On the other hand the energy spread becomes biggest at the optimum matching condition. As shown in Fig. 2 low energy tail is a main cause to increase the energy spread at the current of $11.5 \mathrm{~A}$.

Table 1: Transmission rate, energy and energy spread

\begin{tabular}{|c|c|c|c|c|c|}
\hline $\begin{array}{c}\text { Alpha } \\
\text { Magnet } \\
\text { Current } \\
{[\mathrm{A}]}\end{array}$ & $\begin{array}{c}\text { BCM\#1 } \\
{[\mathrm{mA}]}\end{array}$ & $\begin{array}{c}\text { BCM\#3 } \\
{[\mathrm{mA}]}\end{array}$ & $\begin{array}{c}\text { Transm } \\
-i s s i o n \\
{[\%]}\end{array}$ & $\begin{array}{c}\text { Energy } \\
{[\mathrm{MeV}]}\end{array}$ & $\begin{array}{c}\text { Energy } \\
\text { Spread } \\
{[ \pm \%]}\end{array}$ \\
\hline 7 & 209 & 97 & 46.4 & 57.3 & 0.67 \\
\hline 11.5 & 94 & 68 & 72.3 & 59.5 & 1.16 \\
\hline 12.5 & 57 & 34 & 59.6 & 62.3 & 0.94 \\
\hline
\end{tabular}

The low energy tail originates from the difference of the cavity RF-filling time in the RF-gun and accelerating column. The cavity RF-filling times of the RF-gun and the accelerating column are $0.21 \mu \mathrm{s}$ and $0.83 \mu \mathrm{s}$, respectively. However the effect of low energy tail becomes smaller at the current of $7 \mathrm{~A}$ and $12.5 \mathrm{~A}$. A momentum filter with a fixed gap is on the maximum dispersion point of the trajectory in the alpha magnet. The energy of electron moving on the centre line of the trajectory is $0.9 \mathrm{MeV}$ at the current setting of $11.5 \mathrm{~A}$. At the current of $7 \mathrm{~A}$, the median energy is $0.5 \mathrm{MeV}$, thus the momentum of the head of one macro pulse of electron beam, which is about $0.6 \mu \mathrm{s}$ long, is too small to pass through the following accelerating column. It is possible to reduce energy spread by kicking out the head of one macro pulse of electron beam with a magnetic kicker.

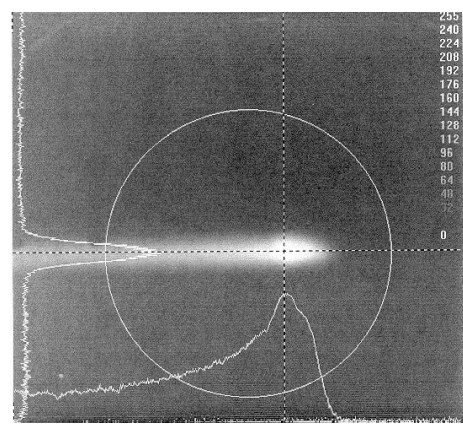

Figure 2: Horizontal and vertical beam profile of the profile monitor after beam analysing magnet at the alpha magnet current setting of 11.5 A. Horizontal profile represents a beam energy spread.

\subsection{Bunch Length Measurement}

The bunch lengths of the electron beams were measured using optical transition radiation with a streak camera of Hamamatsu Photonics Co. The bunch trains are shown in Fig. 3. The measured bunch length is $11.7 \mathrm{ps}$ in FWHM before the RF phase adjustment. After the fine adjustment of RF phase to two accelerating columns, the bunch length reduces to 7.4 ps (FWHM).
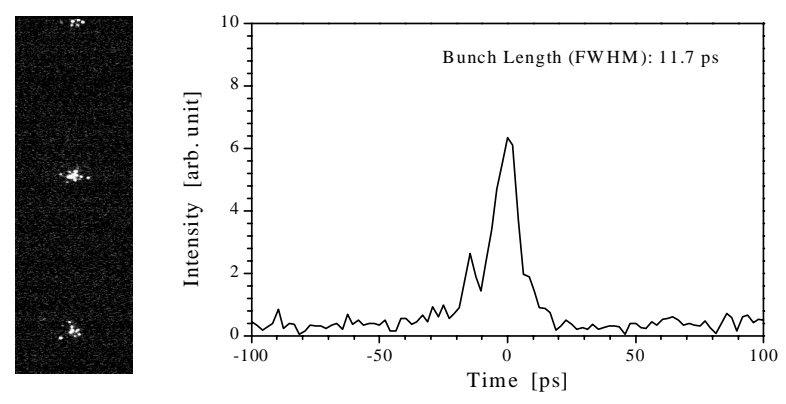

(a)
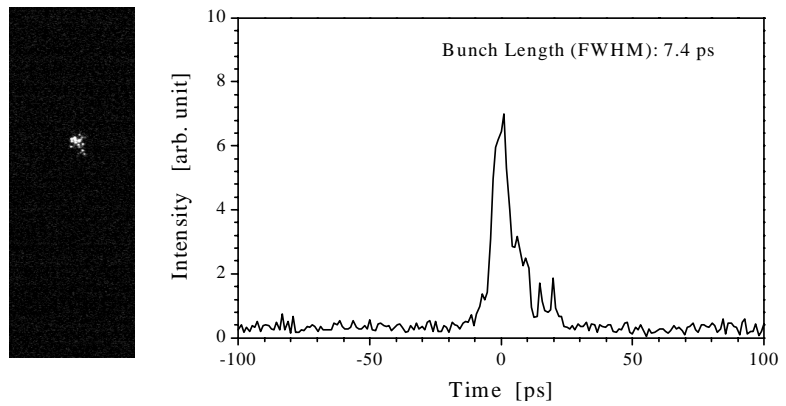

(b)

Figure 3: Streak camera images and its intensity profiles at the alpha magnet current setting of $11.5 \mathrm{~A}$; (a) before and (b) after RF phase adjustment. Vertical lengths of camera images correspond to streak time spans of $1 \mathrm{~ns}$ for (a) and $500 \mathrm{ps}$ for (b). 
The variations of bunch lengths were measured as the alpha magnet current setting changed. It is observed that the bunch length decreases to $4.5 \mathrm{ps}$ as the alpha magnet current is raised, as shown in Fig. 4. This means that electron beam which exits from the RF-gun with higher momentum gets a better bunch compression in the alpha magnet transport line. Figure 5 describes a bunch length shortening during the beam transport in the alpha magnet. In this Figure the free space distance from the RF-gun to the first accelerating column is 95 $\mathrm{cm}$ and the energies of the head and tail particle of one micro bunch are assumed to be $1 \mathrm{MeV}$ and $0.8 \mathrm{MeV}$, respectively. The resultant minimum time difference depends on the alpha magnet current setting. When the alpha magnet current is raised, the beam current at the linac end decreases down remarkably. In order to obtain a sufficient beam current the RF power to RF-gun should be raised.

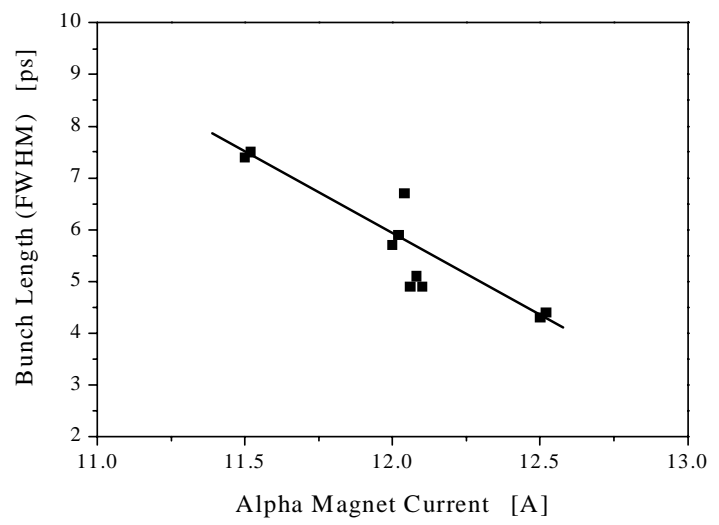

Figure 4: Dependence of bunch length on alpha magnet field gradient.

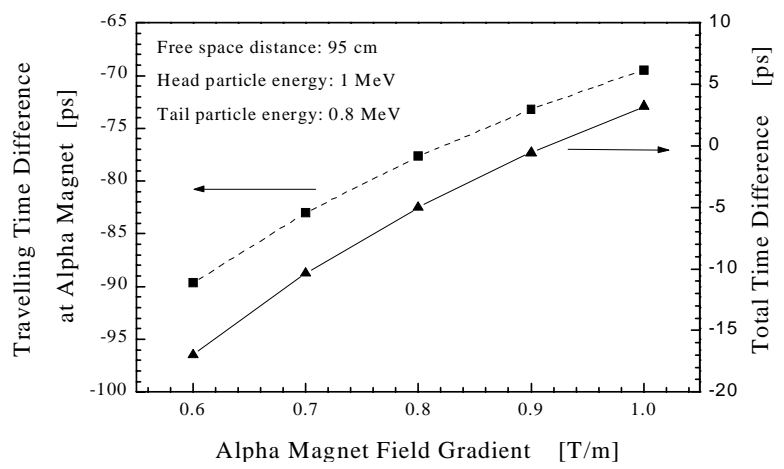

Figure 5: Travelling time difference of head and tail particle at the alpha magnet and total time difference including the travelling time in free space.
The bunches in a few $\mu$ s-long macro pulse of electron beam experience a different accelerating field due to transient beam loading, especially in the thermionic RFgun cavity. The length of each bunch in one macro pulse was measured by changing the trigger time to streak camera. The measurement result is shown in Fig. 6. The bunch length of the end part becomes slightly longer than the head of macro pulse. This increase might be partially caused by unevenness of RF power flattop during one macro pulse. The main cause is transient beam loading in the thermionic RF-gun cavity together with cavity temperature change due to cavity wall dissipation. The effect of transient loading and temperature effect are not fully understood yet and will be investigated in next experiments.

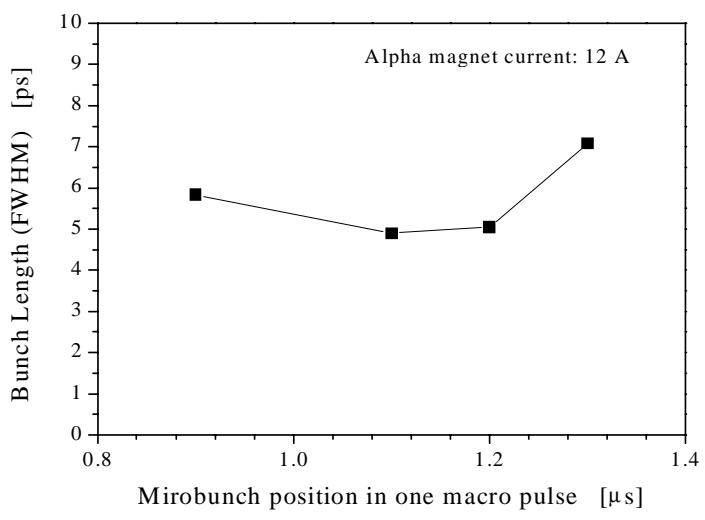

Figure 6: Dependence of bunch length on microbunch position in one macro pulse. Microbunch position represents trigger delay time to streak camera.

\section{SUMMARY}

The beam transmission rate was improved up to $72 \%$ due by the solenoid field focusing and good longitudinal phase matching compared to the previous experiments. Measured energy spread is $\pm 1 \%$ at the beam loading of $100 \mathrm{~mA}$, and the large energy spread at the optimum matching condition is due to cavity RF-filling time differences. The measured bunch length is about $7 \mathrm{ps}$ in FWHM, and decreases to less than 4.5 ps as the alpha magnet current increases. But reduction in the beam current is a problem to solve. The length of each bunch depends on the position in one macro pulse which has a relation to transient beam loading in active RF cavity.

\section{REFERENCES}

[1] Richard L. Sheffield, Proc. 1989 IEEE Particle Accelerator Conf. p. 1098.

[2] H. S. Kang, et al., Proc. 1998 Asian Particle Accelerator Conf.

[3] H. S. Kang, et al., IEEE Trans. Nucl. Sci. 44, No. 4, 1639 (1997). 